\title{
Nonlinear Elliptic Partial Difference Equations on Graphs
}

\author{
John M. Neuberger
}

\section{CONTENTS}

\section{Introduction}

2. Existence of Solutions

3. Variational Algorithms

4. Examples

5. Conclusion

Acknowledgments

References
2000 AMS Subject Classification: 05C50，35A05，35A15，49M15, 58J55, 58J70, 65K10, 90C47

Keywords: superlinear, sign-changing solution, variational method, graphs, GNGA, mountain pass, bifurcation, symmetry
This article furthers the study of nonlinear elliptic partial difference equations (PdE) on graphs. We seek solutions $u: V \rightarrow \mathbb{R}$ to the semilinear elliptic partial difference equation $-L u+f(u)=$ 0 on a graph $G=(V, E)$, where $L$ is the (negative) Laplacian on the graph $G$. We extend techniques used to prove existence theorems and derive numerical algorithms for the partial differential equation (PDE) $\Delta u+f(u)=0$. In particular, we prove the existence of sign-changing solutions and solutions with symmetry in the superlinear case. Developing variants of the mountain pass, modified mountain pass, and gradient Newton-Galerkin algorithms for our discrete nonlinear problem, we compute and describe many solutions. Letting $f=f(\lambda, u)$, we construct bifurcation diagrams and relate the results to the developed theory.

\section{INTRODUCTION}

This paper considers nonlinear partial difference equations (PdE) on graphs. In particular, we prove existence, nodal structure, and symmetry theorems and develop new algorithms for semilinear elliptic PdE. Our efforts parallel recent advances in the study of related partial differential equations (PDE). The survey article [Neuberger 97a] summarizes many of the most relevant PDE results and provides a list of open problems in that area. $\mathrm{PdE}$ are generated whenever PDE are discretized via a grid, which is reason enough to be interested in their solution, but not all PdE arise from PDE. One expects the study of PdE to be as rich, varied, and deep as the study of graph theory, symmetry, and PDE, and the analysis and algorithms used to investigate those fields.

Let $G=(V, E)$ be a simple connected graph, with $m=|V|$ and $n=|E|$ finite. For our numerical experiments, we typically take $f: \mathbb{R} \rightarrow \mathbb{R}$ to be defined by $f(s)=s^{3}+\lambda s$, where $\lambda$ is a real parameter. Our theorems are generalized to the much wider class of superlinear nonlinearities considered in [Ambrosetti and Rabinowitz 73] and [Castro et al. 97a]; in particular, $f$ need 
not be odd. For this example, the primitive $F: \mathbb{R} \rightarrow \mathbb{R}$ is $F(s)=\int_{0}^{s} f(t) d t=\frac{1}{4} s^{4}+\frac{1}{2} \lambda s^{2}$. Arbitrarily, we make a choice of orientation for $G$ in order to define $D \in M_{n \times m}$ so that for an edge $e_{k}=\left(v_{i}, v_{j}\right) \in E$ we have $D_{k i}=-1$, $D_{k j}=+1$, and $D_{k p}=0$ for $p \notin\{i, j\}$. Then independently of any choice of orientation, we define the (negative) Laplacian $L: \mathbb{R}^{m} \rightarrow \mathbb{R}^{m}$ to be the linear map represented by the matrix $L=D^{T} D \in M_{m \times m}$. Numbering our vertices $V=\left\{v_{1}, \ldots, v_{m}\right\}$ and taking $d_{i}$ to be the degree of the $i$ th vertex, we see that $L_{i i}=d_{i}$ and $L_{i j}=-1$ if $\left(v_{i}, v_{j}\right) \in E$ or $\left(v_{j}, v_{i}\right) \in E$, and $L_{i k}=0$ otherwise. Identifying $\mathbb{R}^{m}$ with the set of real-valued functions with domain $V$, we use the terms "function" and "vector" interchangeably and seek $u \in \mathbb{R}^{m}$ satisfying

$$
-L u+f(u)=0 .
$$

Our study of the finite-dimensional semilinear elliptic PdE (1-1) closely follows the related works concerning the PDE

$$
\begin{aligned}
\Delta u+f(u) & =0 \text { in } \Omega, \\
\frac{\partial u}{\partial \eta} & =0 \text { in } \partial \Omega,
\end{aligned}
$$

as well as the similar zero-Dirichlet problem.

The linear operator $L$ has been the object of intense study and much is known about its spectrum. One of the first articles on the subject and an excellent introduction is [Bapat 96]. Additional references are [Chung 97], where an alternative definition of the Laplacian on graphs results in a bounded spectrum, and [Biggs 93], whose definition coincides with ours. Most of the PdE literature concerns linear problems and/or positive solutions, e.g., works by S. S. Cheng, S. T. Liu, C. V. Pao, and G. Zhang (see [Cheng 00, Cheng 99, Cheng and Lin 99, Cheng and Liu 97, Cheng and Pao 02, Liu and Chen 04, Liu et al. 00, Pao 95, Zhang and Cheng 02], and references therein). The papers [Zhang and Cheng 06] and [Zhang 05] consider very similar problems to ours, wherein analytic existence results are provided for onesign and sign-changing solutions to certain nonlinear elliptic PdE for graphs corresponding to regular grids. In general (but not always), nontrivial solutions of (1-1) are saddle points of (1-4), whereby neither minimization nor maximization suffices. As in PDE, the method of supersub solutions seems best suited for obtaining positive solutions. There are some monotone iteration schemes that can be used for approximating sign-changing solutions, but their application to nonlinear elliptic PdE appears to be unconsidered at this point. We seek sign-changing solutions (and information about the symmetry of these solutions) for nonlinear problems. The closest works appear to consider PdE that arise from the regular discretization process required in approximating solutions to PDE via finite differences. See also [Ambrosetti and Rabinowitz 04], [Marchuk 82], and [Strikwerda 89] for more on finite difference schemes for PDE that generate PdE. In particular, [Marchuk 82] discusses variationaldifference techniques. Many of the cited PdE papers consider linear difference operators that are not quite the Laplacian on a graph. Our results could easily apply to nonlinear problems using instead those operators, by first finding a basis of the correspondingly different eigenfunctions using, say, ARPACK (see [Lehoucq et al. 90] and Section 3 of this paper). Many other interesting papers consider related PDE, PdE, and even ODE on graphs; for example, [Pokornyı̆ and Pryadiev 04] presents a system of conventional ODE on a network, treating the equations at the vertices in a novel way. The article [Kevrekidis et al. 02] contains an example related to the time-dependent PdE found in our concluding section. None of the cited references appear to consider symmetry of nonlinear PdE solutions in conjunction with their nodal structure, and very few of the references use methods inspired by established PDE variational techniques. A thorough review of the literature together with the new ideas of this paper will undoubtedly lead to many additional noteworthy results. In particular, alternative boundary conditions and weighted Laplacians should be a fruitful area for future research.

It is well known that there is a natural zero-Neumann boundary condition enforced on solutions to the eigenvalue problem

$$
-L u+\lambda u=0 .
$$

This condition also applies to (1-1). The eigenvalues $\left\{\lambda_{i}\right\}_{i=1, \ldots, m}$ satisfy $\lambda_{1}=0<\lambda_{2} \leq \cdots \leq \lambda_{m}$; we take $\left\{\psi_{i}\right\}_{i=1, \ldots, m}$ to be the corresponding eigenvectors, chosen to be orthonormal with respect to the standard Euclidean norm $\|u\|=\sqrt{u \cdot u}$ in $\mathbb{R}^{m}$. The constant eigenvector with eigenvalue 0 can be taken to be $\psi_{1}=\left(\frac{1}{\sqrt{m}}, \ldots, \frac{1}{\sqrt{m}}\right)$.

In [Castro et al. 97a], a variant of the mountain pass lemma (MPL) is used to duplicate the one-sign existence results of [Ambrosetti and Rabinowitz 73], and then extended to prove the existence of a sign-changing exactlyonce solution. We apply those ideas to our elliptic difference equation. In special cases, we can prove the existence of solutions via more elementary techniques. In [Neuberger 97b], we developed an algorithm for finding certain low-energy solutions of equations such as 
(1-2) using the constructive proofs found in [Castro et al. 97a]. The one-sign algorithm (essentially the mountain pass algorithm, MPA, see also [Choi and McKenna 93]) is adapted without difficulty; the sign-changing algorithm (modified mountain pass algorithm, MMPA, see also [Costa et al. 01]) requires a significant modification. This difficulty is paralleled in considering existence proofs of sign-changing solutions to (1-1). In [Neuberger and Swift 01], the gradient Newton-Galerkin algorithm (GNGA) was developed to investigate (1-2) using a basis of eigenfunctions of the corresponding (continuous) linear problem to span a suitably large finite-dimensional subspace. We adapt this algorithm in an obvious way, although for small finite $m$ we can use the entire basis. In the spirit of [Costa et al. 01], we use knowledge of the symmetry group of $G$ to modify our existence theorems and numerical algorithms to obtain solutions with symmetry.

Let $J: \mathbb{R}^{m} \rightarrow \mathbb{R}$ be defined by

$$
\begin{aligned}
J(u) & =\frac{1}{2} D u \cdot D u-\sum_{i=1}^{m} F\left(u_{i}\right) \\
( & \left.=\frac{1}{2} D u \cdot D u-\frac{1}{4} u^{2} \cdot u^{2}-\frac{1}{2} \lambda u \cdot u\right),
\end{aligned}
$$

where given a function $g: \mathbb{R} \rightarrow \mathbb{R}$ and $u \in \mathbb{R}^{m}$, we take the composition $g \circ u$ to mean $g(u)=\left(g\left(u_{1}\right), \ldots, g\left(u_{m}\right)\right)$. It is easy to see that

$$
\begin{aligned}
J^{\prime}(u)(v) & =-(-L u+f(u)) \cdot v \\
( & \left.=D u \cdot D v-u^{3} \cdot v-\lambda u \cdot v\right),
\end{aligned}
$$

so that critical points of $J$ are solutions to (1-1). Figure 1 depicts typical profiles of $J(t u)$ and $J^{\prime}(t u)(t u)$.

The parallels to the variational setting for the continuous problem (1-2) are clear. For example, for the zero-Dirichlet version of (1-2) and under certain assumptions on a subcritical nonlinearity $f$ (see Section 2 ), the functional $J: H_{0}^{1,2}(\Omega) \rightarrow \mathbb{R}$ defined by

$$
J(u)=\int_{\Omega}\left\{\frac{1}{2}|\nabla u|^{2}-F(u)\right\} d x
$$

is $C^{2}$ and has directional derivative

$$
J^{\prime}(u)(v)=\langle\nabla J(u), v\rangle=\int_{\Omega}\{(\nabla u \cdot \nabla v-f(u) v)\} d x
$$

for all $v \in H$. In [Castro et al. 97a] we define the Nehari manifold

$$
S=\left\{u \in \mathbb{R}^{m}-\{0\}: J^{\prime}(u)(u)=0\right\}
$$

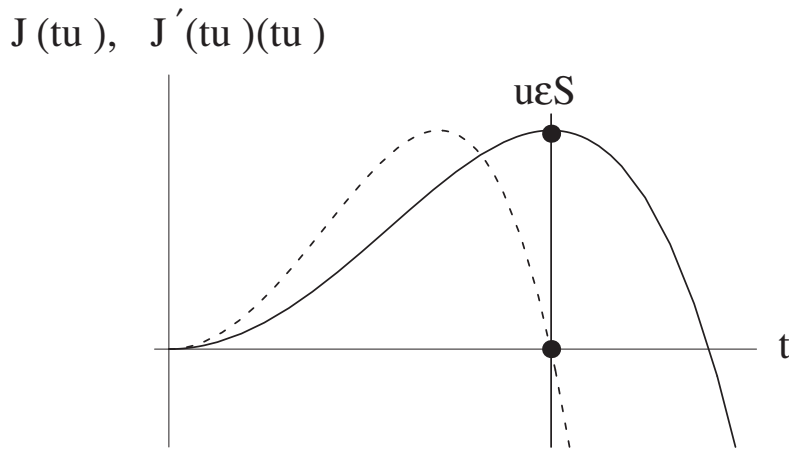

FIGURE 1. The "Volcano" shape of $J$ drives all superlinear elliptic PDE variational arguments. The same holds for our difference equations on graphs. This diagram is for a random nonzero element of $\mathbb{R}^{3}$, viewed as a function on the vertices of the complete graph $G=\mathbf{K}_{3}$, but qualitatively one gets the same picture for other graphs or in the continuous case, for any superlinear $f$ with $f(0)=0$ and $f^{\prime}(0)<\lambda_{1}$.

and an important subset of sign-changing elements

$$
S_{1}=\left\{u \in S-\{0\}: J^{\prime}(u)\left(u_{-}-u_{+}\right)=0\right\},
$$

where $u_{+}(x)=\max \{u(x), 0\}$ and $u_{-}(x)=\min \{u(x), 0\}$ for $x \in V$. For the continuous problem, an equivalent definition (and the one found in [Castro et al. 97a]) is

$$
\hat{S}_{1}=\left\{u \in S-\{0\}: u_{+} \in S, u_{-} \in S\right\} .
$$

For our discrete problem, the two definitions of $S_{1}$ are not equivalent. Indeed, the latter set may be empty or at least fail to have the useful properties exploited in [Castro et al. 97a]. For both the discrete and continuous problems, $u \in S_{1}$ implies that

$$
J(u)\left(u_{-}-u_{+}\right)=J^{\prime}(u)\left(u_{-}\right)-J^{\prime}(u)\left(u_{+}\right)=0
$$

and

$$
J^{\prime}(u)(u)=J^{\prime}(u)\left(u_{-}\right)+J^{\prime}(u)\left(u_{+}\right)=0,
$$

whence $J^{\prime}(u)\left(u_{-}\right)=J^{\prime}(u)\left(u_{+}\right)=0$. For our discrete problem and in light of $(2-4)$, for $u \in S$ (regardless of whether $u \in S_{1}$ ),

$0=J^{\prime}(u)\left(u_{ \pm}\right)=J^{\prime}\left(u_{ \pm}\right)\left(u_{ \pm}\right)+L u_{ \pm} \cdot u_{\mp} \geq J^{\prime}\left(u_{ \pm}\right)\left(u_{ \pm}\right)$,

whence we have $J^{\prime}\left(u_{-}\right)\left(u_{-}\right)=J^{\prime}\left(u_{+}\right)\left(u_{+}\right)$yet we may not have $J^{\prime}\left(u_{-}\right)\left(u_{-}\right)=J^{\prime}\left(u_{+}\right)\left(u_{+}\right)=0$. That is, in contrast to the continuous case, $u \in S_{1}$ does not imply that $u_{ \pm} \in S$. In any case, we will use $(1-7)$ in our efforts to find sign-changing solutions to $\operatorname{PdE}(1-1)$. See Section 2 for discussions of the behavior of $J$ acting on or near these sets and the exact hypothesis on $f$ leading to these properties. We will see that $S$ is a manifold 
(and in fact closed, bounded, and hence compact in this finite-dimensional case). In [Castro et al. 97a], for the superlinear problem and if $f^{\prime}(0)<\lambda_{1}$, we have the following theorem:

Theorem 1.1. There exist positive and negative solutions to (1-2) that are local minimizers of $\left.J\right|_{S}$, and a sign-changing exactly-once solution that is a minimizer of $\left.J\right|_{S_{1}}$.

In that work and here, nonzero functions have a unique ray projection onto $S$, i.e., given $u \neq 0$ there exists a unique $\alpha>0$ such that $\alpha u \in S$. For convenience, we refer to this sign-changing exactly-once solution as the CCN solution.

Generalizations of our previous theories and algorithms to nonsuperlinear problems have been moderately successful (see for example [Castro et al. 97b, Castro et al. 99, Castro et al. 03, Neuberger 98]). One might expect that task to be somewhat easier in the finite-dimensional setting of this paper.

Understanding the implications of symmetry is essential in our investigations. In this paper, we demonstrate where such knowledge is useful, leaving deeper graphtheoretic applications and discoveries for subsequent efforts. In particular, consideration of the innovations of [Neuberger et al. 05a] and [Neuberger et al. 05b] will be of immediate benefit to follow-up research done in this new setting of nonlinear elliptic PdE on graphs.

The paper is organized as follows. In Section 2 we state and prove existence, nodal structure, and symmetry theorems. These results generally follow our prior semilinear elliptic PDE arguments, taking care to incorporate our new definition for $S_{1}(1-7)$ and to deal with the difficulties implied by Lemma 2.6. In Section 3 we provide some basic variational formulations and the numerical algorithms used in our experimental investigations. These algorithms are variants of the GNGA (see [Neuberger and Swift 01, Neuberger et al. 03, Neuberger et al. 05b]), the MPA (see [Choi and McKenna 93] and [Neuberger 97b]), and the MMPA (see [Costa et al. 01, Cossio et al. 00, Neuberger 97b, Neuberger 98]). Many of these iterative schemes were inspired by constructive proofs, while others provided the insight that led to new proofs. In Section 4 we analyze several examples of lower-order graphs, chiefly the complete graph $\mathbf{K}_{3}$. In particular, we introduce ideas for investigating the symmetry of solutions. We close the section with some results from new algorithms that suggested the techniques of proof used in Section 2. Finally, we provide a section containing some concluding remarks and results from considering linear parabolic and hyperbolic PdE.

\section{EXISTENCE OF SOLUTIONS}

We wish to emphasize that many of the elements of the theorem statements and proofs found in this section were understood only after certain key numerical experiments were designed and performed. In particular, the new definition for $S_{1}(1-7)$ was a consequence of nearly successful but ultimately failed numerical simulations using the previous definition found in (1-8). Diagnosing the problem in these experiments led to the statement and proof of Lemma 2.6, which led the way to the proofs as one now sees them. Thus, we find that the sections following this one that contain variational equations, algorithms, and experimental results are particularly interesting and relevant.

The proofs in this section hold for a more general class of nonlinearity than those typically used in our numerical experiments. We assume essentially the same hypothesis found in [Ambrosetti and Rabinowitz 73, Castro et al. 97a, Choi and McKenna 93, Rabinowitz 86]. In particular, we take $f \in C^{1}(\mathbb{R}, \mathbb{R})$ such that $f(0)=0$ and the following conditions hold. Our key assumption is that $f$ is superlinear, i.e.,

$$
\lim _{|u| \rightarrow \infty} \frac{f(u)}{u}=\infty
$$

We also make use of the convexity assumption

$$
f^{\prime}(u)>\frac{f(u)}{u} \quad \text { for } u \neq 0 .
$$

We assume that $f^{\prime}(0)<\lambda_{1}=0$, although as in [Castro et al. 03] it is almost ensured that one could relax this condition to $f^{\prime}(0)<\lambda_{2}$ and still obtain sign-changing solutions (the proofs in that paper deal with technical difficulties due to the lack of compactness and infinitedimensionality that are not likely to arise here).

In the continuous case (1-2) one must make additional assumptions. For completeness we include them here. In [Castro et al. 97a], we additionally assume that there exists $m \in(0,1)$ such that

$$
\frac{m}{2} f(u) u \geq F(u)
$$

(in fact this need hold only for $|u|>\rho$ for some $\rho>0$ ). This condition implies the coercivity of $J$ on $S$ and is used to make up for the loss of compactness in proving convergence of minimizing sequences. Analysis of the PDE also 
requires that we assume that there exist constants $A>0$ and $p \in\left(1, \frac{N+2}{N-2}\right)$ such that $\left|f^{\prime}(u)\right| \leq A\left(|u|^{p-1}+1\right)$ for all $u \in \mathbb{R}$. It follows that $f$ is subcritical, i.e., there exists $B>0$ such that $|f(u)| \leq B\left(|u|^{p}+1\right)$. For $N=1$ this condition is omitted, while for $N=2$ it suffices to have $p \in(1, \infty)$ (see [Ambrosetti and Rabinowitz 73]). In our finite-dimensional setting the coercive and subcritical assumptions appear unnecessary, but might come in handy if one attempted to get a "convergence result," i.e., look at a family of graphs increasing in order and try to say something about a continuous problem that was being approximated.

We are not concerned with loss of compactness, subcritical growth, or the Sobolev embedding theorem in our finite-dimensional setting. Clearly our functional $J$ is well defined and twice differentiable on all of $\mathbb{R}^{m}$. In this section and under the above hypotheses, we state and prove the following theorems.

Let $f$ be a superlinear function, not necessarily odd, with $f(0)=0$ and $f^{\prime}(0)<\lambda_{1}=0$. Then for a connected finite graph $G$ we have the following results:

Theorem 2.1. There exist positive and negative solutions to (1-1) that are local minimizers of $\left.J\right|_{S}$.

Theorem 2.2. There exists a solution to (1-1) that is a global maximizer of $\left.J\right|_{S}$.

We say that a function on $G$ changes sign exactly once if the subgraphs induced by $\{v \in V: u(v)>0\}$ and $\{v \in V: u(v)<0\}$ are connected.

Theorem 2.3. There exists a solution to (1-1) that changes sign exactly once and is a minimizer of $\left.J\right|_{S_{1}}$.

If $m \geq 3$, we can demonstrate that there exists a solution (distinct from the minimizer) that is a maximizer of $\left.J\right|_{S_{1}}$. It seems true that the maximizer of $\left.J\right|_{S_{1}}$ and $\left.J\right|_{S}$ are one and the same, but we do not have a proof of that fact.

Let $\mathbf{Q}$ be the symmetry group of $G$. If $f$ is odd, define $\mathcal{Q}=\mathbf{Q} \times \mathbf{Z}_{2}$; otherwise, take $\mathcal{Q}=\mathbf{Q}$. The possible symmetries of solutions correspond to conjugacy classes of maximal isotropy stabilizer subgroups of $\mathcal{Q}$ (see [Neuberger et al. 05b] for more details). Let $\chi$ be a fixed-point symmetry subspace of $\mathbb{R}^{m}$ corresponding to a given symmetry, that is, there exists a subgroup in such a conjugacy class whose elements fix $\chi$ pointwise. Then we also have the following:
Theorem 2.4. There exists a solution to (1-1) that is a minimizer of $\left.J\right|_{S \cap \chi}$. If $\operatorname{dim}(S \cap \chi) \geq 1$, then there exists a second (distinct) solution that is a maximizer of $\left.J\right|_{S \cap \chi}$.

It has not been proved that $S_{1}$ is a manifold. If it were, and low-dimensional numerical experiments indicate that it can be, the sign-changing proof could be simplified. Nevertheless, we still have the following:

Theorem 2.5. Given a fixed-point symmetry subspace $\chi$ such that for all $u \in \chi$ with $u_{+}, u_{-} \neq 0$ we have $u_{+}, u_{-} \in$ $\chi$, there exists a solution to (1-1) that is a minimizer of $\left.J\right|_{S_{1} \cap \chi}$. Given a fixed-point subspace $\chi$ such that $u \in \chi$ implies $u_{+}, u_{-} \neq 0$, there exists a solution to (1-1) that is a minimizer of $\left.J\right|_{S \cap \chi}$.

Apparently, all possible sign-changing solutions fall into one of the two cases. Again, if $\operatorname{dim}\left(S_{1} \cap \chi\right) \geq 1$, we can demonstrate that there exists a second (distinct) solution that is a maximizer of $\left.J\right|_{S_{1} \cap \chi}$. It seems true that the maximizers of $\left.J\right|_{S_{1} \cap \chi}$ and $\left.J\right|_{S \cap \chi}$ are one and the same.

The ability for us to distinguish two solutions as two distinct solutions will depend to some extent on the application. For instance, some solutions will belong to two different fixed-point subspaces $\chi_{A}$ and $\chi_{B}$, whereby it cannot be known without additional information that the same solution $u$ does not satisfy $\min _{S \cap \chi_{A}} J=$ $\min _{S \cap \chi_{B}} J=J(u)$. For other fixed-point spaces, it will be clear that $\chi_{A} \cap \chi_{B}=\{0\} \not \subset S$, so that the corresponding minimizers (and maximizers) will be distinct solutions. In Section 4, we present an example in which minimizers and maximizers of $\left.J\right|_{S}, J_{S_{1}}, J_{S \cap \chi}$, and $J_{S_{1} \cap \chi}$ are explicitly and/or numerically found.

We first present a lemma, which represents something of an obstacle toward applying the techniques from [Castro et al. 97a]. We note that in the continuous case, the equivalent equation is $\int_{\Omega} \nabla u_{+} \cdot \nabla u_{-} d x=0$, which implies the nice additivity properties $J(u)=J\left(u_{+}\right)+J\left(u_{-}\right)$, $J^{\prime}(u)(u)=J^{\prime}\left(u_{+}\right)\left(u_{+}\right)+J^{\prime}\left(u_{-}\right)\left(u_{-}\right)$, and so on. There are elementary examples of functions on graphs for which these equalities do not hold, i.e., the inequality in the lemma is strict.

Lemma 2.6. Given $u \in \mathbb{R}^{m}$ we have

$$
L u_{+} \cdot u_{-}=L u_{-} \cdot u_{+} \geq 0 .
$$

Proof: Using the notation that $k_{x}$ is the degree of vertex $x \in V$ and that $x_{i}, i=1, \ldots, x_{k}$, are the neighbors of $x$, 
we see that

$$
L u_{+} \cdot u_{-}=\sum_{x \in V}\left\{k_{x} u_{+}(x)-\sum_{i=1}^{k_{x}} u_{+}\left(x_{i}\right)\right\} u_{-}(x) \geq 0
$$

since the only possible nonzero terms are the result of positive neighbors $u_{+}\left(x_{i}\right)$ multiplying the negative value $u_{-}(x)$.

We now state some of the useful properties of the Nehari manifold $S$ defined in (1-6) and of the functional $J$ acting on or near $S$. We take $\lambda<\lambda_{1}=0$; otherwise, $S$ is degenerate and fails to be a manifold.

1. $S$ is a closed $(m-1)$-dimensional $C^{1}$ manifold in $\mathbb{R}^{m}$.

2. $J^{\prime \prime}(0)(u, u)>0$ for all $u \in \mathbb{R}^{m}, 0 \notin S$, and $J \geq c>0$ on $S$.

3. $S$ is bounded (in contrast to the infinite-dimensional case).

4. $J$ is bounded above on $S$ (in contrast to the infinitedimensional case).

5. Given $u \neq 0 \in \mathbb{R}^{m}$ there exists a unique $\alpha>0$ such that $\alpha u \in S$.

6. For $u \in S, J(u)>J(t u)$ for all $t \in[0, \infty)-\{1\}$ and $J^{\prime \prime}(u)(u, u)<0$.

7. $u$ is a nontrivial solution to (1-1) if and only if $u$ is a critical point of $\left.J\right|_{S}$.

These facts follow from arguments that are virtually identical to those found in [Castro et al. 97a]. The fact that $S$ is closed ( 0 is not a limit point of $S$ ) was claimed in [Castro et al. 97a] and later proved in [Neuberger 98]. In this finite-dimensional setting, this is obvious. That $S$ is a manifold follows from the implicit function theorem. Observe that $S$ is the zero set of $\gamma(u)=J^{\prime}(u)(u)=L u \cdot u-u \cdot f(u)$. Then by $(2-2)$ and for $u \in S$ we have

$$
\begin{aligned}
J^{\prime \prime}(u)(u, u) & =L u \cdot u-u^{2} \cdot f^{\prime}(u)<L u \cdot u-u \cdot f(u) \\
& =\gamma(u)=0 .
\end{aligned}
$$

That is to say, the gradient of $\gamma$ is nonvanishing on its zero set, which is thus a manifold. Note that

$$
J^{\prime \prime}(0)(u, u)=L u \cdot u-f^{\prime}(0) u \cdot u>0
$$

by the Poincaré inequality (or characterization of the smallest eigenvalue $\left.\lambda_{1}=0\right)$. Since 0 is a local minimum and $f$ superlinear implies that for $u \neq 0$ we have
$J(\alpha u) \rightarrow-\infty$ as $\alpha \rightarrow \infty$, we see that there must exist an $\alpha>0$ such that $\alpha u \in S$. This $\alpha$ is unique, since $(2-5)$ says that every critical value in the ray direction must be a maximum. By Lagrange multipliers, $u$ is a nontrivial critical point of $J$ if and only if it is a critical point of $\left.J\right|_{S}$. Indeed, if $u$ is a constrained critical point then $\nabla J(u)=t \nabla \gamma(u)$ for some multiple $t$ of the normal vector to $S, \nabla \gamma(u)$. This implies that $t=0$, and hence $\nabla J(u)=0$, since $(2-5)$ gives $\gamma(u) \cdot u<0$; yet $\nabla J(u) \cdot u=0$ (by virtue of $u \in S$ ). Since $S$ is closed and bounded, hence compact (due to the finite dimension of $\mathbb{R}^{m}$ ), the continuous function $J$ must be bounded on $S$. In this discrete case it is not hard to see that there exists $\delta>0$ such that $J(u) \geq \delta>0$ for all $u \in S$. Some of these facts are less useful than in the continuous case, since we are now dealing with a compact manifold and do not, for example, have to worry whether bounded sequences have only weakly convergent subsequences.

We now prove Theorem 2.1.

Proof: Let $\left\{u_{n}\right\} \subset S$ be a minimizing sequence, i.e., $J\left(u_{n}\right) \downarrow \min _{S} J$. Since $S$ is a compact set, there exists a subsequential limit $u \in S$ satisfying $J(u)=\min _{S} J$. By the above discussions, the constrained minimum is in fact a critical point of $J$, hence a solution to (1-1). Suppose that $u$ were sign-changing. Since $u$ is a solution and hence $J^{\prime}(u)\left(u_{ \pm}\right)=0$, Lemma 2.6 implies that $\gamma\left(u_{ \pm}\right) \leq 0$. Without loss of generality, let $0<c \leq d \leq 1$ be such that $c u_{+}, d u_{-} \in S$. Then

$$
J(u) \geq J(c u) \geq J\left(c u_{+}\right)+J\left(c u_{-}\right)>J\left(c u_{+}\right),
$$

since $\gamma\left(d u_{-}\right)=0$ and $c \leq d$ implies that $J\left(c u_{-}\right)>0$. This is a contradiction, since $c u_{+} \in S$ yet $J(u)=\min _{S} J$. Thus, $u$ is a one-sign solution to $(1-1)$. If $f$ is odd, $-u$ is automatically a critical point of the even functional $J$, hence a one-sign solution of the opposite sign. Suppose that $f$ is not odd and without loss of generality assume that the solution we just found is positive. Then, we can repeat the above argument using $\tilde{f}$ defined by $\tilde{f}(u)=$ $-f(-u)$ for $u>0$ and $\tilde{f}(u)=f(u)$ for $u \leq 0$ to get a pair of one-sign solutions. Since we are using the other branch of $f$, the negative solution is in fact a negative solution to the original problem.

The proof of Theorem 2.2 is almost a corollary.

Proof: Let $\left\{u_{n}\right\} \subset S$ be a maximizing sequence, i.e., $J\left(u_{n}\right) \uparrow \max _{S} J$. Since $S$ is a compact set, there exists a subsequential limit $u \in S$ satisfying $J(u)=\max _{S} J$. By the above discussions, the constrained maximum is in fact a critical point of $J$, hence a solution to (1-1). 
If nondegenerate, the minimizers are of Morse index (MI) 1 and the maximizer is of MI $m$. It seems almost ensured that the maximizer is a sign-changing solution, but we have not proved this. One need show only, to confirm this conjecture, that given $u \in S$ of one sign, there exists $v \in S_{1}$ with $J(u)<J(v)$, which seems likely in light of Figure 6.

We now prove Theorem 2.3.

Proof: As in [Castro et al. 97a], the separation property of $S_{1}$ is key. Accordingly, let $u \in S_{1}$ and consider $z(t)=$ $P_{S}\left((1-t) u_{+}+t u_{-}\right)=\alpha(t)\left((1-t) u_{+}+t u_{-}\right)$, for some smooth function $\alpha:[0,1] \rightarrow(0,2]$. Clearly $\alpha\left(\frac{1}{2}\right)=2$, so that $z\left(\frac{1}{2}\right)=u \in S_{1}$. Now suppose that $\frac{d}{d t}(J(z(t)))=0$ for some $t=t^{*}$. Since $z \in S, 0=J^{\prime}(z)(z)=J^{\prime}(z)\left(z_{+}\right)+$ $J^{\prime}(z)\left(z_{-}\right)$, so that $J^{\prime}(z)\left(u_{-}\right)=\frac{t-1}{t} J^{\prime}(z)\left(u_{+}\right)$. Since

$$
\begin{aligned}
0=\frac{d}{d t}(J(z(t))) & =\frac{\alpha^{\prime}}{\alpha} J^{\prime}(z)(z)+\alpha J^{\prime}(z)\left(u_{-}-u_{+}\right) \\
& =\alpha J^{\prime}(z)\left(u_{-}-u_{+}\right)
\end{aligned}
$$

we have

$$
J^{\prime}(z)\left(u_{-}\right)=J^{\prime}(z)\left(u_{+}\right)=\frac{t-1}{t} J^{\prime}(z)\left(u_{+}\right) .
$$

This implies that $J^{\prime}(z)\left(u_{-}\right)=J^{\prime}(z)\left(u_{+}\right)=0$, hence $\gamma\left(z_{ \pm}\right) \leq 0$, since $\frac{t-1}{t} \neq 1$. By $(2-2)$,

$$
\begin{aligned}
J^{\prime \prime}\left(z_{ \pm}\right)\left(z_{ \pm}, z_{ \pm}\right) & =L z_{ \pm} \cdot z_{ \pm}-z_{ \pm}^{2} \cdot f^{\prime}\left(z_{ \pm}\right) \\
& <L z_{ \pm} \cdot z_{ \pm}-z_{ \pm} \cdot f\left(z_{ \pm}\right)=\gamma\left(z_{ \pm}\right) \leq 0 .
\end{aligned}
$$

Now, using Lemma (2.6) we obtain

$$
\begin{aligned}
\frac{d^{2}}{d t^{2}}(J(z(t)))= & \alpha^{\prime} J^{\prime}(z)\left(u_{-}-u_{+}\right) \\
& +\alpha^{2} J^{\prime \prime}(z)\left(u_{-}-u_{+}, u_{-}-u_{+}\right) \\
= & \alpha^{2} j^{\prime \prime}(z)\left(u_{-}-u_{+}, u_{-}-u_{+}\right) \\
= & \frac{1}{t^{2}} J^{\prime \prime}\left(z_{+}\right)\left(z_{+}, z_{+}\right) \\
& +\frac{1}{(1-t)^{2}} J^{\prime \prime}\left(z_{-}\right)\left(z_{-}, z_{-}\right)-2 \alpha^{2} L u_{+} \cdot u_{-} \\
< & 0 .
\end{aligned}
$$

Hence, the critical point of $J \circ z$ for $t \in(0,1)$ is unique and a maximum, For this value $t^{*}$, we have $J(z)\left(z_{-}-z_{+}\right)=$ 0 and so $u=z\left(t^{*}\right) \in S_{1}$ and $J(u)>J(z(t))$ for all $t \in[0,1]-\left\{\frac{1}{2}\right\}$. In fact, $S_{1}$ separates any $v>0$ and $w<0$ on $S$. Let $z:[0,1] \rightarrow S$ now denote any path on $S$ so that $z(0)=v$ and $z(1)=w$. For $0<t \ll \frac{1}{2}$, one sees that $\gamma(z)=0, \gamma\left(z_{-}\right)>0$, and $\gamma\left(z_{+}\right)<0$ (see Figure 1). Similarly, for $1>t \gg \frac{1}{2}$, we have that $\gamma(z)=0$, $\gamma\left(z_{-}\right)<0$, and $\gamma\left(z_{+}\right)>0$, implying that

$$
J^{\prime}(z)\left(z_{-}-z_{+}\right)=\gamma\left(z_{-}\right)-\gamma\left(z_{+}\right)
$$

changes sign for some $t=t^{*} \in(0,1)$. For $u=z\left(t^{*}\right)$ we have that $J^{\prime}(u)\left(u_{-}-u_{+}\right)=0$, and hence $u \in S_{1}$.

Proceeding as in the one-sign existence proof, we find a minimizer $u \in S_{1}$ satisfying $J(u) \geq J(v)$ for all $v \in S_{1}$. We do not know that $S_{1}$ is a manifold and so cannot apply Lagrange multipliers. However, if we suppose that $u$ is not a solution we can find a contradiction. As in [Castro et al. 97a], take the path $z(t)=P_{S}\left((1-t) u_{+}-t u_{-}\right)$and in a neighborhood about $u$ apply the deformation lemma. As a result, we would follow the negative gradient flow (projected tangent to $S$; we know that a nonzero gradient cannot be orthogonal to $S$ ) and obtain a deformed path that (a) still connects positive to negative elements of $S$ and hence intersects $S_{1}$ by the above separation property, and (b) has a strictly smaller maximum $J$ value along it. This cannot be, since we started the flow with a path through the minimizer of $\left.J\right|_{S_{1}}$. Thus, we have a solution that necessarily changes sign by virtue of membership in $S_{1}$. An argument very similar to the one-sign case shows that the solution must change sign exactly once. If not, we could construct an element of $S_{1}$ with strictly smaller $J$ value, another contradiction.

We conclude with the proofs of Theorems 2.4 and 2.5.

Proof: If $\left\{u_{n}\right\}$ is a minimizing (maximizing) sequence in $S \cap \chi$, then as above we get a subsequential limit. The resulting minimizer (maximizer) $u$ is in $S \cap \chi$. By Lagrange multipliers, we know that $\nabla J(u)$ cannot be nonzero and normal to $S$. By invariance, the gradient lies in $\chi$. Thus, the constrained critical point of $\left.J\right|_{S \cap \chi}$ is a critical point of $J$ and hence a solution to (1-1) with the symmetry type corresponding to the fixed-point subspace $\chi$.

Let $\chi$ be a fixed-point subspace with the property that if $u \in \chi$ with $u_{+}, u_{-} \neq 0$ then also $u_{+}, u_{-} \in \chi$. If $\left\{u_{n}\right\}$ is a minimizing sequence in $S_{1} \cap \chi$, then as above we get a subsequential limit. The resulting minimizer $u$ is in $S_{1} \cap \chi$. Now, the path $z(t)=P_{S}\left((1-t) u_{+}+\right.$ $\left.t u_{-}\right) \in S \cap \chi$ as well, since $u_{+}, u_{-} \in \chi$. Again using the invariance of the gradient, we see that assuming that $\nabla J(u) \neq 0$ leads to a contradiction. This follows from the fact that the deformed path will also lie in $\chi$, so that the separation property of $S_{1}$ yields an element of $S_{1} \cap \chi$ with strictly lower $J$ value than the minimum value $J(u)$. Necessarily, this solution changes sign and is of a symmetry type corresponding to the fixed-point subspace.

Finally, let $\chi$ be a fixed-point subspace with the property that if $u \in \chi$ then $u_{+}, u_{-} \neq 0$. Using above arguments, we get a convergent minimizing sequence of $\left.J\right|_{S \cap \chi}$. Using the symmetry invariance of the gradient, we see 
that the minimizer is a solution without the need to appeal to the deformation lemma. This follows since $S \cap \chi$ is a manifold. In hindsight, this solution belongs to $S_{1}$ since it is a solution that changes sign. It is of a symmetry type corresponding to the fixed-point subspace.

All of the above proofs can be seen in action by studying the simple example $G=\mathbf{K}_{3}$ in Section 4. For example, consider the sign-changing branch of symmetry type $B$ corresponding to $\psi_{3}$. Any sign-changing vector $u$ of the form $(b, b, a)$ (without loss of generality assume that $b<0)$ has $u_{+}=(0,0, a)$ and $u_{-}=(b, b, 0)$ of type $B$ as well. Thus, the paths on $S$ connecting $\alpha u_{+} \in S$ to $\alpha u_{-} \in S$ (which must pass through $S_{1}$ ) are composed of elements also of type $B$. Minimization in $S_{1} \cap \chi_{B}$ necessarily results in a sign-changing solution of symmetry type $B$. Additionally, consider the type $D$ branch that also bifurcates from $\lambda_{2}=\lambda_{3}=3$. It is not true that elements of this symmetry type have positive and negative parts of the same symmetry type, but by virtue of the symmetry type, such elements are already signchanging if nontrivial. Thus, minimization in $S \cap \chi_{D}$ results in a sign-changing solution of symmetry type $D$. In both cases for this low-dimensional example we in fact obtained isolated points on intersecting an invariant subspace with either $S$ or $S_{1}$. Thus, minimizing sequences are constant in $\chi_{B} \cap S_{1} \neq \emptyset$ and $\chi_{D} \cap S \neq \emptyset$, since $\operatorname{dim}\left(\chi_{B} \cap S_{1}\right)=0$ and $\operatorname{dim}\left(\chi_{D} \cap S\right)=0$.

As a final comment, there is much that could be proved concerning bifurcation. Our automated code in [Neuberger et al. 05b] relies on developed theory of symmetry, fixed-point subspaces, and bifurcation (see for example [Golubitsky et al. 88]). The equivariant branching lemma (EBL) is perhaps the core tool we use to predict bifurcation. The EBL is useful to ponder when one is writing code to find new bifurcation branches, and should prove equally useful in proving theorems concerning the existence of such branches. The EBL implies the "bifurcation from simple eigenvalues" results used so heavily by nonlinear functional analysts studying variational functionals for elliptic PDE (see for example [Rabinowitz $86])$.

\section{VARIATIONAL ALGORITHMS}

We began our foray into this relatively new field by extending GNGA to investigate problem (1-1). For more complicated graphs of higher order, finding the canonical basis of eigenfunctions may entail modification of the
ARPACK code found in [Neuberger et al. 05a], and definitely will require extending and automating the symmetry analysis and branch-following techniques found in [Neuberger et al. 05a] and [Neuberger et al. 05b].

We assume that $u=\sum_{i=1}^{m} c_{i} \psi_{i}$ and seek coefficients $c \in \mathbb{R}^{m}$ such that the coefficient vector of the standard gradient's eigenfunction expansion $g=$ $\left(J^{\prime}(u)\left(\psi_{i}\right)\right)_{i=1, \ldots, m}$ is zero. Note that

$$
\begin{aligned}
g_{i} & =D u \cdot D \psi_{i}-f(u) \cdot \psi_{i}=\left(L \sum c_{j} \psi_{j}\right) \cdot \psi_{i}-f(u) \cdot \psi_{i} \\
& =c_{i} \lambda_{i}-f(u) \cdot \psi_{i} .
\end{aligned}
$$

For small $m$, the equivalent expression $g_{i}=L u$. $\psi_{i}-\psi_{i} \cdot f(u)$ can be computed efficiently without reference to the eigenfunction expansion coefficients $c_{i}$. Similarly, the Hessian $h \in M_{m \times m}$ defined by $h=$ $\left(J^{\prime \prime}(u)\left(\psi_{i}, \psi_{j}\right)\right)_{i, j=1, \ldots, m}$ can be computed as

$$
\begin{aligned}
h_{i j} & =D \psi_{i} \cdot D \psi_{j}-f^{\prime}(u) \psi_{i} \cdot \psi_{j} \\
& =\lambda_{i} \delta_{i j}-f^{\prime}(u) \psi_{i} \cdot \psi_{j},
\end{aligned}
$$

where $\delta_{i j}$ is the Kronecker delta. Applying Newton's method with step size $\delta \in(0,1]$ to find zeros of $g$ results in our algorithm:

1. Initialize $c=c^{0} \in \mathbb{R}^{m}$ and set $u=u^{0}=\sum c_{i} \psi_{i}$.

2. Loop until $\|\nabla J(u)\|=\sqrt{g \cdot g}$ is small:

(a) Compute the gradient vector $g \in \mathbb{R}^{m}$.

(b) Compute the Hessian matrix $h \in M_{m \times m}$.

(c) Solve for the search direction $\chi$ that satisfies $h \chi=g$.

(d) Step $c=c^{k+1}=c^{k}-\delta \chi$.

(e) Update $u=u^{k+1}=\sum c_{i} \psi_{i}$.

(f) Output data, compute norm of gradient.

The output data can vary depending on the experiment; typical choices include the value of $J\left(u^{k}\right)$, calculated similarly to the gradient and Hessian, and the signature $\operatorname{sig}\left(u^{k}\right)$, which we take to be the number of negative eigenvalues of $h=h\left(u^{k}\right)$. If $u$ is a nondegenerate solution to $(1-1)$, then $\operatorname{sig}(u)$ equals the Morse index (MI) of $u$. The MI can be thought of as the number of "down" directions of the critical point, e.g., $\mathrm{MI}=0$ for minima, $\mathrm{MI}=m$ for maxima, and $\mathrm{MI} \in\{1, \ldots, m-1\}$ for saddle points in between. The search direction $\chi$ can be solved using any number of linear solvers, even dealing with possibly noninvertible Hessians $h$. Noninvertible Hessians inevitably occur at boundaries of basins of attraction of 
Newton's method, fractal when taking discrete steps, and at degenerate critical points. This is good news actually, since the first situation can be avoided by knowing good guesses and the second can lead to interesting symmetrybreaking bifurcations.

We also present a few experimental results relating to adaptations of the MPA and MMPA from [Neuberger 97b]. In part, we do so because their operation exposes the existence theory. In [Castro et al. 97a] and [Neuberger $97 \mathrm{~b}$, we see that given $u \neq 0$ there exists $\alpha>0$ such that $\alpha u \in S$. This holds true for our current (discrete) problem as well. In [Castro et al. 97a] and [Neuberger $97 \mathrm{~b}]$, one sees that $S_{1}$ has the property that given a sign-changing function $u$ there exist $\alpha, \beta>0$ such that $\alpha u_{+} \in S, \beta u_{-} \in S$, and as a result, $\alpha u_{+}+\beta u_{-} \in S_{1}$. For our current (discrete) problem, we see that given a sign-changing function $u$ there exist $\alpha>0$ and $t \in(0,1)$ such that with $z$ defined by $z=\alpha\left((1-t) u_{+}+t u_{-}\right)$, we have $J^{\prime}(z)\left(z_{-}-z_{+}\right)=0$ and hence $z \in S_{1}$. Combining these constraints with Sobolev gradient steepest descent (see also [Neuberger 97c]) results in the MPA for finding MI 1 one-sign solutions and the MMPA for finding MI 2 sign-changing solutions of (1-2). In the PDE case, using the "correct" Hilbert space and the associated gradient leads to good numerical performance; one may view the resulting search direction as a preconditioned version of the poorly performing (and only densely defined) $L^{2}$ gradient. The "Sobolev gradient" appears to perform in a similarly advantageous fashion when one is seeking critical points of the action functional for finite-dimensional PdE as well. The brief pseudocode is as follows:

1. Choose $u=u_{0}$ as a one-sign element of the function space.

2. Project $u$ onto $S$ by doing steepest ascent in the ray direction.

3. Compute an approximation of $\nabla J(u)$ by solving an appropriate linear system.

4. Loop until the approximation of $\nabla J(u)$ is small:

(a) Take the gradient descent step: $u_{k+1}=u_{k}-$ $\delta \nabla J\left(u_{k}\right)$.

(b) Project $u$ onto $S$ by doing a steepest ascent in the ray direction.

(c) Compute an approximation of $\nabla J(u)$ by solving the linear system.

Here, $\delta \in(0,1]$ is the step size, and the linear system in question (for the continuous zero-Dirichlet problem
$(1-2))$ satisfies $-\Delta(\nabla J(u))=\nabla_{2} J(u)$, where $\nabla_{2} J(u)$ is the "usual" Euclidean gradient. Borrowing from the method used in [Cossio et al. 00], we can construct a Sobolev gradient for our discrete Neumann problem that has the good performance indicative of using the proper norm (see Figure 5). Defining $g_{i}$ as in (3-1), we obtain the Sobolev gradient

$$
\nabla_{S} J(u)=g_{1} \psi_{1}+\sum_{i=2}^{m} \frac{g_{i}}{\lambda_{i}} \psi_{i}
$$

The MMPA requires one to start with a sign-changing initial guess and to project iterates onto $S_{1}$ as opposed to $S$. For the continuous problem, this can be accomplished by

$$
P_{S_{1}}(u)=\alpha u_{+}+\beta u_{-},
$$

where $\alpha, \beta \in(0, \infty)$ are chosen such that $\alpha u_{+}=P_{S}\left(u_{+}\right)$ and $\beta u_{-}=P_{S}\left(u_{-}\right)$are on $S$. Projecting functions onto $S$ is just steepest ascent in the ray direction (see Figure 1 and Section 2). Briefly, starting with $u_{0}=u$ and iterating

$$
u_{k+1}=u_{k}+\delta \frac{J^{\prime}\left(u_{k}\right)\left(u_{k}\right)}{\left|u_{k}\right|^{2}} u_{k}
$$

results in convergence to $P_{S}(u)$. The term $J^{\prime}\left(u_{k}\right)\left(u_{k}\right)$ can be approximated in several ways of varying degrees of numerical complexity, efficiency, and accuracy, including using the eigenfunction expansion ideas from [Cossio et al. 00]. For our discrete problems, the projections of iterates onto $S_{1}$ must be accomplished via a different method. We saw in Section 2 that given a sign-changing vector $u \in \mathbb{R}^{m}$, the path

$$
\begin{aligned}
z(t) & =P_{S}(r(t))=P_{S}\left((1-t) u_{+}+t u_{-}\right) \\
& =\alpha(t)\left((1-t) u_{+}+t u_{-}\right)
\end{aligned}
$$

has essentially the same properties as it did in the continuous case. We see that

$$
\begin{aligned}
\frac{d}{d t} J(z) & =J^{\prime}(z)\left(\alpha^{\prime} r+\alpha r^{\prime}\right) \\
& =\frac{\alpha^{\prime}}{\alpha} J^{\prime}(z)(z)+\alpha J^{\prime}(z)\left(u_{-}-u_{+}\right) \\
& =\alpha J^{\prime}(z)\left(u_{-}-u_{+}\right)
\end{aligned}
$$

is zero only for some unique $t^{*} \in(0,1)$ such that $J\left(z\left(t^{*}\right)\right)>J(z(t))$ for all $t \in[0,1]-\left\{t^{*}\right\}$, and that in fact $z\left(t^{*}\right) \in S_{1}$ as defined in (1-7) (see Figure 6). If $u \in S_{1}$, then $t^{*}=\frac{1}{2}$ and $\alpha\left(t^{*}\right)=2$. Thus, given a sign-changing vector $u$ we take gradient ascent steps in the $u_{-}-u_{+}$direction and project iterates onto $S$, until the maximum value is achieved and we are on $S_{1}$. For example, the 
Mathematica fragment for effecting these projections (efficient enough for small problems) that uses the built in secant method is as follows:

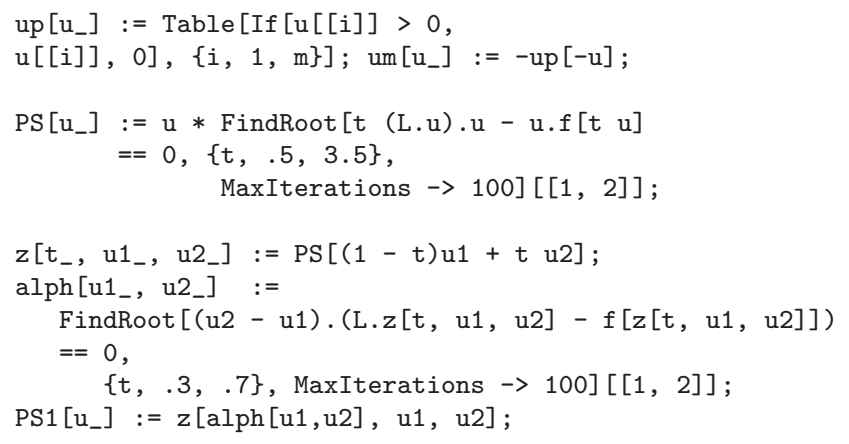

We will employ algorithms that are further variants of the MPA and MMPA. First, since we are in a finitedimensional setting, we can do steepest ascent and find critical points (solutions to (1-1)) that are maximizers. This is easily accomplished by replacing

$$
u_{k+1}=u_{k}-\delta \nabla J\left(u_{k}\right)
$$

with

$$
u_{k+1}=u_{k}+\delta \nabla J\left(u_{k}\right) .
$$

Second, we borrow from ideas in [Costa et al. 01] and [Neuberger et al. 05b] and restrict our optimization to invariant subspaces corresponding to specified symmetries. For example, when seeking a solution vector in $\mathbb{R}^{3}$ when $G$ is the complete graph $\mathbf{K}_{3}$, we might want to restrict our search to elements of the form $(b, b, a)$ in an invariant subspace $\chi_{B}$ (see the $\mathbf{K}_{3}$ example in Section 4). Given a vector $u=\left(b_{1}, b_{2}, a\right)$, we execute the projection

$$
P_{\chi_{B}}(u)=\left(\frac{b_{1}+b_{2}}{2}, \frac{b_{1}+b_{2}}{2}, a\right)
$$

after each gradient step. In theory, the gradient $\nabla J(u)$ is invariant under the group actions corresponding to the chosen symmetry, but in practice, small computational errors may lead to instability. The projection $u_{k}=P_{S} P_{\chi_{B}} \hat{u}_{k}$, for example, will ensure that the iterate $u_{k}$ is an element of the manifold that maintains the symmetry of type $B$. Other symmetry types corresponding to other subgroups can be similarly enforced.

\section{EXAMPLES}

In this section we demonstrate the numerical and analytical techniques by looking at a relatively simple example, namely the complete graph $\mathbf{K}_{3}$. We are able to prove some extra facts in this concrete case, but more importantly we show the inner workings of each algorithm and theorem. We take a thorough approach in looking at this problem, with an eye toward testing our new algorithms and gaining insight into the structure needed to prove existence and nodal structure theorems.

Our initial experiments on regular square grids were entirely analogous to those found in [Neuberger and Swift 01]. As noted in the introduction, discretizing a PDE leads to a PdE. We do not report here further on these executions other than to note that it is enlightening to be reminded that using numerical algorithms to solve PDE are really attempts to solve discrete problems exactly.

In this paper, our goal is to further develop techniques for studying nonlinear elliptic PdE on graphs. By understanding the underlying symmetries of a given graph $G$, one should be able to choose a useful order for the basis of eigenfunctions of $L$ for $\mathbb{R}^{m}$. This is an essential step for understanding the expected proliferation of symmetric solutions, aiding in both our numerical investigations and subsequent efforts to find existence and nodal structure proofs. Adapting the ARPACK code (see [Neuberger et al. 05a]) and automated branch-following methods (see [Neuberger et al. 05b]) that we have so successfully used on continuous problems, we will then be able to thoroughly investigate very large graphs with large numbers of symmetries. Applying the sophisticated approach taken in [Neuberger et al. 05a] and [Neuberger et al. 05b] (where the $\mathbf{D}_{6} \times \mathbf{Z}_{2}$ symmetry of the hexagon (Koch's snowflake) is exploited) will be a fruitful area for several reasons. Applying those concepts, the symmetry of the graph (and hence the basis) can be used by an automated program that follows the trivial branch, makes a turn at each zero eigenvalue (bifurcation point), and continues making turns at each secondary (or tertiary) bifurcation point. At each turn this automated code uses the critical eigenfunctions of the Hessian to perturb off of the parent branch. This approach is particularly interesting at multiple critical eigenvalues, where algorithmic knowledge of the symmetry of the basis (and hence possible symmetries of solutions) is required in order to follow all possible types of (conjugacy classes of) branches. This is achieved by applying knowledge of the structure of the symmetry group of the graph $G$. Future developments in the field of nonlinear elliptic PdE on graphs will be obtained by considering the methods and ideas in [Neuberger et al. 05a] and [Neuberger et al. 05b], where in theory a single push of a button may almost completely generate an accurate and informative bifurcation diagram annotated with a plethora of relevant information concerning exis- 
tence, multiplicity, symmetry, nodal structure, MI, and so on.

\section{1 $\mathrm{K}_{3}$}

Our main example demonstrates that for simple graphs one can work out the existence and "nodal structure" of some solutions exactly, while unexpected complexities result in a structure rich enough to provide secondary bifurcations and solutions not easily come by analytically. Let $G=\mathbf{K}_{3}$ be the complete graph with three degree-2 vertices. There is in some sense a maximal amount of symmetry to exploit in complete graphs.

Due to the superlinear nature of the nonlinearity $f$, the graph of $J$ for $\lambda<\lambda_{1}=0$ is a "volcano," e.g., 0 is a local minimum, each ray intersects the rim (the manifold $S$ ) once, and so on. Indeed, these properties drive all of the existence proofs in [Castro et al. 97a] and many related works. Figure 1 shows the graphs of $J(t u)$ and $J^{\prime}(t u)(t u)$ for a randomly selected nonzero vector in $\mathbb{R}^{3}$.

In initial experiments, we used the basis of eigenfunctions of $L$ for $\mathbb{R}^{3}$ that was automatically provided by Mathematica. Subsequently, we found an alternative basis that contains eigenfunctions of each symmetry type to be a more convenient choice, although this may not be possible for every type of graph. The matter of choosing a "proper" basis in general has not been settled.

For this elementary example, by plugging $u=c \psi_{i}$ into (1-1) one easily obtains complete information about several branches. For example, the trivial branch is $c \equiv 0$, and the one-sign branch $u \equiv c$ bifurcates to the left from $\lambda_{1}=0$ with $c^{2}=-\lambda$ and $J(c u)=\lambda^{2}$. The conjugacy class of the permutations of $u=c(-1,1,0)$ has branches bifurcating to the left from $\lambda_{2}=\lambda_{3}=3$, where $c^{2}=3-\lambda$ and $J(c u)=\frac{1}{2}(3-\lambda)^{2}$. It is not so easy to work out in closed form the results for solutions that are not exact multiples of eigenfunctions. In this supposedly simple example, there are secondary bifurcations. The secondary bifurcation point on the one-sign branch can be easily found exactly for any complete graph. These secondary solutions are not multiples of an eigenfunction; an algorithm such as GNGA or one of the modified MPAtype algorithms seems to be required to investigate such branches.

To demonstrate the symmetry arguments from [Neuberger et al. 05a] and [Neuberger et al. 05b] to which we have alluded, we more carefully analyzed the symmetry of eigenfunctions and solutions. The symmetry group for $G$ is $\mathbf{D}_{3}$, that is, six rotations and flips. Allowing for sign changing antireflections, our group expands to $\mathbf{D}_{3} \times \mathbf{Z}_{2} \simeq \mathbf{D}_{6}$. Forming the 16 subgroups of $\mathbf{D}_{6}$ and

\begin{tabular}{|c|c|c|c|}
\hline \# Classes & Symm. Type & Elements & Dim Invar. Subspace \\
\hline \hline 1 & $A$ & $(a, b, c)$ & $\operatorname{dim}\left(\chi_{A} \cap S\right)=2$ \\
\hline 3 & $B$ & $\begin{array}{c}(b, b, a) \\
(a, b, b) \\
(b, a, b)\end{array}$ & $\begin{array}{c}\operatorname{dim}\left(\chi_{B_{i}} \cap S\right)=1 \\
i=1,2,3\end{array}$ \\
\hline 1 & $C$ & $(a, a, a)$ & $\operatorname{dim}\left(\chi_{C} \cap S\right)=0$ \\
\hline 3 & $D$ & $\begin{array}{c}(-b, b, 0) \\
(0,-b, b) \\
(b, 0,-b)\end{array}$ & $\begin{array}{c}\operatorname{dim}\left(\chi_{D_{i}} \cap S\right)=0 \\
i=1,2,3\end{array}$ \\
\hline 1 & $E$ & $(0,0,0)$ & $\chi_{E} \cap S=\emptyset$ \\
\hline
\end{tabular}

TABLE 1. For $\mathbf{K}_{3}$, the manifold $S$ is 2-dimensional. The intersection of the invariant subspaces with $S$ is of one lower dimension than the invariant subspaces themselves. All solutions belong to one or more of the appropriate intersections. From the ordering in (4-1), we see that $\chi_{E} \subset \chi_{C} \subset$ $\chi_{B_{i}} \subset \chi_{A}$ and $\chi_{E} \subset \chi_{D_{i}} \subset \chi_{A}$.

translating back to $\mathbf{D}_{3} \times \mathbf{Z}_{2}$, we see that there are five symmetry types of solutions. These are the five conjugacy classes of maximal stabilizer (isotropy) subgroups. A representative of a symmetry class corresponds to a subspace that is invariant under the actions of that subgroup. The symmetry types can be partially ordered as

$$
A \prec B \prec C \prec E \text { and } A \prec D \prec E \text {, }
$$

where, for example, $B \prec C$ means that if $H \in B$ and $K \in C$ then $H$ is a subgroup of $K$. See Table 1 for a summary of the invariant subspaces corresponding to these five symmetry types.

Here $A$ is the symmetry that corresponds to the subgroup containing only the identity. Solutions of this type have no symmetry. Type $B$ is the symmetry type of the flip, i.e., each element of the class fixes a function of the form $(b, a, b)$ (or one of its permutations) on $G=\mathbf{K}_{3}$. Symmetry type $C$ fixes only the constant functions, i.e., invariance under both rotations and flips. Symmetry type $E$ corresponds to the whole symmetry group and fixes only the trivial solution $u=0 \in \mathbb{R}^{m}$. Finally, symmetry type $D$ corresponds to invariance under antiflips, e.g., functions of the form $(b, 0,-b)$ (or one of its permutations) on $G=\mathbf{K}_{3}$. A natural goal for future efforts will be to systematize and automate the construction of the hierarchy (lattice) of symmetry types for each new graph investigated. In Figure 3, the primary branch of constant solutions (multiples of $\psi_{1}$ ) is of symmetry type $C$, invariant under all rotations and flips. The only subgroups of the subgroup of type $C$ are of symmetry types $B$ and $A$, which have invariance under flips, and only the identity, respectively. Thus, the only possible bifurcations at $\lambda=-\frac{3}{2}$ off of the constant branch are of symmetry types $B$ and $A$. The displayed secondary branch is of symmetry type $B$, with eigenvector expansion coefficients of the 


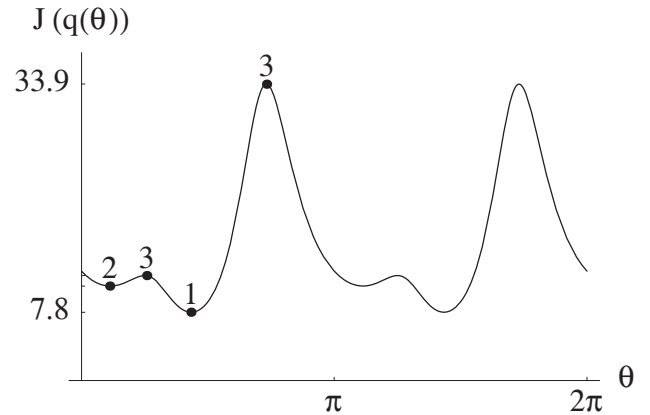

FIGURE 2. Graph of the functional $J$ restricted to the 1dimensional set obtained by intersecting a type- $B$ invariant subspace for $\mathbf{K}_{3}$ with the manifold $S$. Extremal points are solutions. See Figure 3 to view the locations of these solutions on the bifurcation diagram depicting all symmetry type $B$ solutions.

form $\left(c_{1}, 0, c_{3}\right)$, indicating that added to the type- $C \psi_{1^{-}}$ component is some amount of the type- $B \psi_{3}$-component $\frac{1}{\sqrt{6}}(-1,-1,2)$.

Armed with this symmetry information, the somewhat trial and error process suggested by Figure 4 is perhaps unnecessary although still informative. For example, without using the deeper implications of the equivariant branching lemma (see [Golubitsky et al. 88]) and considering higher-order derivatives of $J$, we cannot be certain that we have not missed an asymmetric branch (type $A$ ) bifurcating from the constant solution branch at this location. Since the extrema in Figure 4 are all of symmetry type $C$, we can be fairly confident, how-

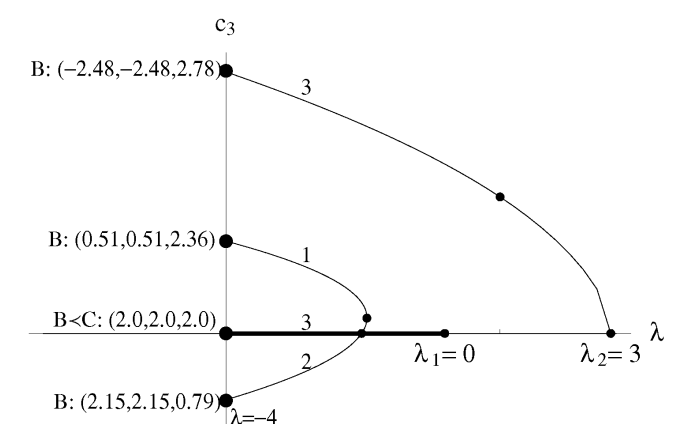

FIGURE 3. All symmetry type $B$ solutions to (1-1) when $G=\mathbf{K}_{3}$ can be found in this diagram. Each nonconstant branch corresponds to 6 solutions, plus-or-minus from each of the 3 conjugacy classes. The small dots indicate bifurcation points where the Hessian is singular. The 4 large dots denote solutions located on the graph in Figure 2; approximate values of the solutions at $\lambda=-4$ are given. The thick line on the $\lambda$ axis is the constant solution bifurcating to the left from $\lambda=0$. The $\lambda$ axis also corresponds to the trivial type $E$ solution, although this is not an element of $\chi_{B} \cap S$ since $0 \notin S$.

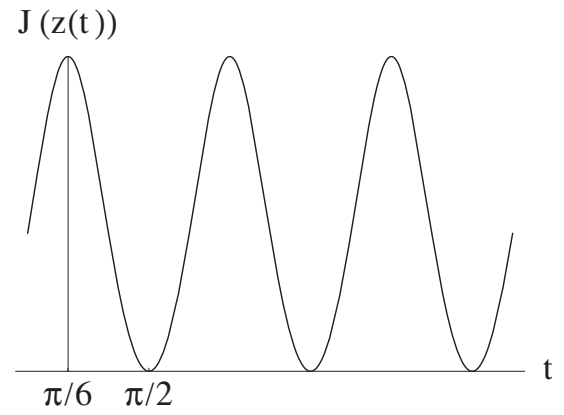

FIGURE 4. A method for finding perturbation directions at a bifurcation point. Here, $z(t)=\alpha(t)\left(u^{*}+\cos (t) \psi_{2}+\right.$ $\sin (t) \psi_{3}$ ), where $u^{*}$ is the one-sign solution (multiple of constant eigenfunction $\psi_{1}$ ) at $\lambda^{*}=-\frac{3}{2}, \lambda=-\frac{7}{4}$ is just to the left of the bifurcation point, and $\alpha=\alpha(t)$ is chosen such that $z \in S$ for all $t \in(0,2 \pi)$. The 3 minima correspond to points that are reasonably likely to be in the basin of attraction of MI 1 solutions, while the maxima should lead toward MI 2 solutions. That $\psi_{2}$ and $\psi_{3}$ span the critical eigenspace follows from analyzing the Hessian at the critical bifurcation value in the $\mathbf{K}_{3}$ case presented here.

ever. The following experiments using Sobolev gradient descent and ascent restricted to invariant subspaces (see also Figure 2) give further evidence that there is no such missing branch.

We have found three different types of solutions of symmetry type $B$ at $\lambda=-4$. There are eighteen altogether, since each type has three rotations (the type- $B$ conjugacy class has three subgroup elements) and the negative of each solution is a solution. Let $\chi_{B}$ be an invariant subspace corresponding to one of these conjugacy classes, say the one with elements of the form $(b, b, a)$. Intersecting this 2-dimensional subspace with the Nehari manifold $S$ results in a 1-dimensional submanifold containing all solutions of symmetry type $B$ from this conjugacy class. Minimizers and maximizers of $J$ restricted to this submanifold must be solutions of symmetry type $B$ or $C$, since $B \prec C$ implies that $\chi_{C} \subset \chi_{B}$ and the trivial type $E$ solution is not an element of $S$. Let $q(\theta)=P_{S}(\cos (\theta), \cos (\theta), \sin (\theta))$. Then the range of $q$ is precisely the 1-dimensional manifold $\chi_{B} \cap S$. Figure 2 shows the graph of $J \circ q$, with four of the eight extremal points and their corresponding MI noted. These points are solutions to (1-1) that have either symmetry type $B$, or in the case of the constant solutions, symmetry type $C$. The solutions are also noted on the portion of the bifurcation diagram found in Figure 3. It is interesting to note that minimizers in this invariant subspace can be of either MI 1 or MI 2; all we know is that there is at least one more "down direction" by virtue of being an element of $S$. 
The modifications of the MPA and MMPA obtained by restricting to invariant subspaces work well (keep in mind that $f^{\prime}(0)=\lambda<\lambda_{1}=0$ must be assumed for $S$ to be a manifold). In many low-dimensional cases, the invariant subspace has a 0 - or 1-dimensional intersection with $S$ or $S_{1}$. The former leads to a solution in one iteration, while the latter efficiently leads to convergence by performing what amounts to 1-dimensional steepest descent and/or ascent. The four featured solutions noted in Figures 2 and 3 were all separately located using the MPA modified to perform steepest descent and/or ascent in $\chi_{B}$ (see $(3-5) ; B=B_{1}$ corresponds to the conjugacy class with symmetry $(b, b, a))$. Such results are easily verified, since plugging a solution vector into (1-1) is a simple matter. Although we have not proved that $S_{1}$ is a manifold, it appears from our experiments that it is when $G=\mathbf{K}_{3}$. If so, clearly it is of dimension 1 . We can see that $\chi_{B} \cap S_{1}$ is not empty, since letting $u=(-1,-1,2)$ we have $u, u_{+}, u_{-} \in \chi_{B}$, and hence $z(t)=P_{S}\left((1-t) u_{+}+\right.$ $\left.t u_{-}\right) \in \chi_{B} \cap S_{1}$ for all $t \in[0,1]$. Thus, $\chi_{B} \cap S_{1}$ is zerodimensional, in fact containing precisely two points, the solution $z\left(t^{*}\right) \in S_{1}$ obtained by maximizing $J \circ z$ on $[0,1]$, and the "antipodal" solution gained by replacing $u$ with $-u,(1,1,-2)$.

\subsection{MPA and MMPA}

In preparation for looking for existence proofs of the type found in [Castro et al. 97a], we looked for low-energy solutions using the MPA and a modified version of the MMPA. The above $\mathbf{K}_{3}$ symmetry-type- $B$ experiments used the MPA, modified as noted in Section 3, by applying the projection equation $(3-5)$. The Sobolev gradient can be easily computed (see (3-3)). Using purposefully bad initial guesses, MPA converged to the one-sign (constant) solution in about seven iterations, from which one can infer that the appropriate space, norm, and hence gradient were used. The plot on the left in Figure 5 depicts the norm of the gradient as a function of MPA iteration number for this execution.

We indicated in Section 3 that we must use the alternative definition of $S_{1}$ found in (1-7). The difficulty is quite interesting. In [Castro et al. 97a], and as a result in [Neuberger $97 \mathrm{~b}$, we rely heavily on the fact that since $u_{+}$ and $u_{-}$are disjoint, then $J(u)=J\left(u_{+}\right)+J\left(u_{-}\right)$. It also follows that $J^{\prime}(u)\left(u_{+}\right)=J^{\prime}\left(u_{+}\right)\left(u_{+}\right)$, so that $u_{+}, u_{-} \in S$ implies that $u \in S_{1}$. None of these statements is true for graphs; in general $L u_{+} \cdot u_{-} \neq 0$. Our alternative definition for $S_{1}$ makes the MMPA work. Once we had proved that the new set has many of the same properties as found in [Castro et al. 97a] (see Section 2), we were able
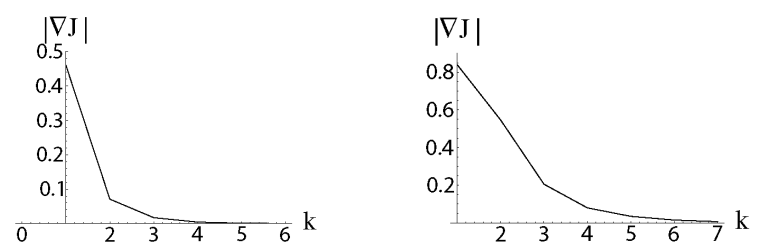

FIGURE 5. Convergence of MPA to the constant one-sign and the modified MMPA to the sign-changing exactly-once solutions of (1-1) for $G=\mathbf{K}_{3}$ at $\lambda=-\frac{1}{2}$. Despite starting with intentionally bad initial guesses, both algorithms yield solutions accurate to 4 or 5 decimal places in $k=7$ iterations.

to prove the existence of a sign-changing exactly-once solution. Figure 6 suggests that, like the explicit construction in [Castro et al. 97a], along the convex combination projected onto $S$ connecting positive and negative parts of a sign-changing function $u, J$ achieves its maximum at or near the midpoint. We see that every path on $S$ connecting a positive function to a negative function will intersect $S_{1}$. Using the initial guess from Figure 6 , the MMPA converged to the CCN MI 2 minimal-energy signchanging exactly-once solution (see also Figure 5).

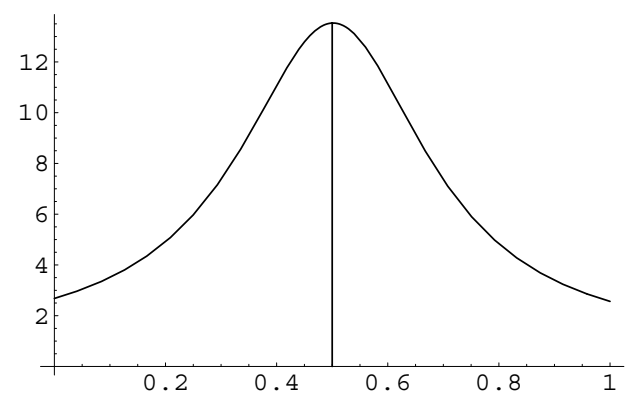

FIGURE 6. This plot of $J(z(t))=J\left(P_{S}(r(t))\right)=J\left(P_{S}((1-\right.$ $\left.t) u_{+}+u_{-}\right)$) could have easily come from a continuous problem, although it was in fact generated using a specific signchanging vector $u \in \mathbf{R}^{3}$ while studying $G=\mathbf{K}_{3}$. In this example, $u$ satisfied $u, c u_{+}, c u_{-} \in S$ for some $c \in(0,1)$. Noticing that the maximum occurred close to but not exactly at $t=\frac{1}{2}$ lead to the alternative definition (1-7). If $u \in S_{1}$, then the maximizer would occur at precisely $t=\frac{1}{2}$.

\subsection{Other Graphs and General Results}

We considered other graphs, although we do not plan to report much of the findings in this paper. In fact, our first GNGA experiments were on the circulant graph $G=\mathbf{C}_{13}(1,3,4)$, which has $m=13$ vertices and $n=39$ edges. The eigenvalues of $L$ are approximately

$$
\sigma=\{0,4.69722, \ldots, 4.69722,8.30278, \ldots, 8.30278\}
$$


with two multiplicity-6 eigenvalues. These values can be in theory calculated using algebraic equations. We computed the various symmetry types of eigenfunctions (vectors in $\mathbb{R}^{m}$ ), six for each of the two distinct multiple eigenvalues. See Figure 7 for a plot of a pair of MI 2 CCN solutions to $(1-1)$ for $\mathbf{C}_{13}(1,3,4)$. We also did experiments on $\mathbf{K}_{4}$ and obtained complete results paralleling the $\mathbf{K}_{3}$ results presented above. The simple experiments done on $\mathbf{K}_{3}$ and the resulting complexities should be enough to convince the reader that when considering a general graph $G$, it will first be necessary to thoroughly understand the implications of the graph's symmetries, order a basis, and automate the branch-following code.
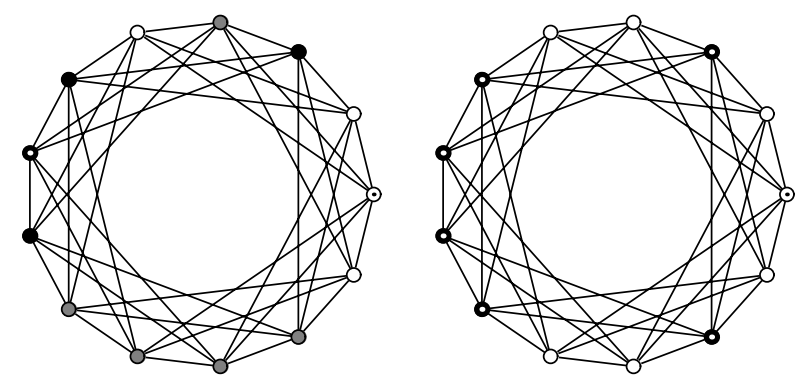

FIGURE 7. "Contour plots" of a pair of MI 2 sign-changing solutions to (1-1) for $G=\mathbf{C}_{13}(1,3,4)$. Black corresponds to positive values, gray to zero values, and white to negative values; small opposite color dots indicate where a extremal values are obtained. The two solutions are of distinct symmetry type. These CCN solutions were obtain using the MMPA, modified for graphs, as described in this paper.

We contemplated what can be said in general about nonlinear elliptic difference equations on complete graphs. It is not difficult to see that the complete graph $\mathbf{K}_{m}$ has a simply described set of eigenvectors. On the one hand, $\mathbf{K}_{m}$ is fairly simple and well studied; on the other, it has a large symmetry group. As we first saw when analyzing the $\mathbf{K}_{3}$ problem, we can show that there exists and describe completely the constant branch bifurcating from $\lambda_{1}=0$ and do the same for the conjugacy class of permutations of $\Psi_{2}$ (since it has only \pm 1 and 0 entries). When $G$ is a complete graph a straightforward calculation computes the Hessian matrix at $\lambda=-\frac{m}{2}$ and shows that there is a multiplicity- $(m-1)$ zero eigenvalue and hence a bifurcation point with a jump from MI 1 to MI $m$ at $\lambda=-\frac{m}{2}$ as one travels left along the constant branch starting from $\lambda_{1}=0$. It might be worthwhile to work out the rest of the details concerning superlinear elliptic PdE on complete graphs.

In Section 2, we proved the existence of one-sign and sign-changing solutions, in general and of specified sym- metry. The proofs were inspired by the work in [Castro et al. 97a], but many of the required techniques came to light only after the variants of the MPA and MMPA were correctly stated, coded, and tested. In particular, the correct definition of $S_{1}$ found in (1-7) was discovered during the consideration of certain experimental failures. Finally, we tested the MMPA (and other algorithms) for several nonodd nonlinearities. In our theorems we do not assume oddness; it was gratifying to see that the algorithms still converged to the expected solutions.

\section{CONCLUSION}

Open questions and areas for future inquiry in partial difference equations on graphs abound. Extending the representation theory found in [Neuberger et al. 05a] to analyze symmetry types of eigenvectors for the discrete Laplacian is an obvious first step. Not only is this of interest in its own right, but it is an essential step toward understanding all possible solutions to nonlinear elliptic equations. It is not clear that there exists in all cases a "canonical" basis; perhaps orthogonality and symmetry types do not always go hand in hand. Calculating the conjugacy classes of the symmetry group as done here and in [Neuberger et al. 05b] for larger graphs with varying degrees of symmetry is the next step, since this will enable the implementation of automated branch following where decisions based on symmetry aid in following bifurcating branches at multiply degenerate bifurcation points. In future efforts, we may use the code recently developed by the author's colleague N. Sieben using GAP (Graphs, Algorithms, and Programming; see http://www.gap-system.org/gap/) to automate the process of cataloging symmetry types and ordering the basis. For large graphs, the ARPACK code of [Neuberger et al. 05a] or perhaps the parallel version PARPACK will be necessary in order to efficiently compute the eigenpairs. We are interested in embedding graphs into metric spaces, whereby one might be able to approximate PDE on manifolds using ideas from this paper. Weighted Laplacians could be used. This might be interesting in itself, or, if the weights are chosen depending on the location of vertices in a metric space, then again approximations to PDE might be obtained. It may be necessary to use Monte Carlo integration methods to form the gradients and Hessians when investigating elliptic PDE on high-dimensional regions. This suggests that perhaps the same random distribution of points could define a random graph, and the corresponding (weighted?) Laplacian might be used to generate a matching basis. Applications in statistical mechanics, computer science, and 
mathematical biology are known to exist (see cited references and references therein for a start). Further investigations into such applications will be interesting.

Graphs of all types of symmetry abound; it has been suggested that the Peterson graph and the Gray semisymmetric (bipartite) graph will be interesting. We ran experiments on nonsymmetric (and much less symmetric) graphs analogous to the experiments with small complete graphs detailed above. In particular, we wondered whether a small graph with simple eigenvalues would be free of secondary bifurcations. The answer is "not necessarily": We found secondary bifurcations when investigating a nonsymmetric graph with five vertices. A systematic approach is likely to be fruitful, although perhaps no more (or less) conclusive than the typical foray into questions of classification in graph theory.

The GNGA amounts to a simple application of Newton's method on $K: \mathbb{R}^{m} \rightarrow \mathbb{R}^{m}$ defined by $K u=$ $-L u+f(u)$, but uses the symmetry of the graph and solutions of an associated linear problem to yield a sufficient understanding of the basins of attraction. Clearly the large amount of known theory related to graphs and their Laplacians will be useful in the future research of nonlinear PdE.

One can consider types of PdE other than elliptic (see [Kevrekidis et al. 02] for an example in which discrete nonlinear time-dependent equations are considered). Any PDE with a " $-\Delta$ " in it could be converted to a PdE using Laplacians on graphs. In fact, derivatives of any order can be replaced with an appropriate difference matrix. As our first foray in to these other areas, we considered the following parabolic and hyperbolic equations:

$$
\begin{aligned}
u_{t} & =-L u, \\
u(0) & =u_{0}=\sum_{i=1}^{m} a_{i} \psi_{i},
\end{aligned}
$$

and

$$
\begin{aligned}
u_{t t} & =-L u, \\
u(0) & =u_{0}=\sum_{i=1}^{m} a_{i} \psi_{i}, \\
u^{\prime}(0) & =v_{0}=\sum_{i=2}^{m} b_{i} \psi_{i} \quad\left(v_{0} \cdot \mathbf{1}=0 \Longrightarrow b_{1}=0\right),
\end{aligned}
$$

where one seeks solutions $u:(0, \infty) \rightarrow \mathbb{R}^{m}$, again identifying $\mathbb{R}^{m}$ with functions mapping $V$ to $\mathbb{R}$. These elementary first- and second-order linear systems can be solved in a straightforward way using separation of variables. Respectively, the solutions can be written as

$$
u(t)=\sum_{i=1}^{m} a_{i} e^{-\lambda_{i} t} \psi_{i}=\left(e^{-t L}\right) u_{0}
$$

and

$$
\begin{aligned}
u(t) & =\sum_{i=1}^{m} a_{i} \cos \left(\lambda_{i}^{1 / 2} t\right) \psi_{i}+\sum_{i=2}^{m}\left(b_{i} / \lambda_{i}^{1 / 2}\right) \sin \left(\lambda_{i}^{1 / 2} t\right) \psi_{i}, \\
& =\cos \left(t L^{1 / 2}\right) u_{0}+L^{\dagger^{1 / 2}} \sin \left(t L^{1 / 2}\right) v_{0}
\end{aligned}
$$

where $\nmid$ denotes a pseudoinverse. Numerical experiments using explicit and implicit methods generated satisfactory approximations to the above solutions. Considering the extension of these nonelliptic equations to nonlinear situations, equations of mixed type, the inclusion of other-order difference terms, and complicated graphs should suggest many directions for further mathematical inquiry.

\section{ACKNOWLEDGMENTS}

The author thanks Nandor Sieben, Jim Swift, and Steve Wilson, of Northern Arizona University, for their help in suggesting graphs and explaining the associated symmetries. The articles [Neuberger et al. 05a] and [Neuberger et al. 05b] contain a much more detailed explanation of the symmetry arguments found in Figure 3.

This work was partially supported by NSF grant DMS0074326

\section{REFERENCES}

[Ambrosetti and Rabinowitz 73] A. Ambrosetti and P. Rabinowitz. "Dual Variational Methods in Critical Point Theory and Applications." J. Functional Analysis 14 (1973), 349-381.

[Ambrosetti and Rabinowitz 04] A. Ashyralyev and P. Sobolevskii. "New Difference Schemes for Partial Differential Equations." In Operator Theory: Advances and Applications, 148. Basel: Birkhäuser Verlag, 2004.

[Bapat 96] R. B. Bapat. "The Laplacian Matrix of a Graph." Math. Student 65:1-4 (1996), 214-223.

[Biggs 93] N. Biggs. Algebraic Graph Theory. Second edition. Cambridge, UK: Cambridge University Press, 1993.

[Castro et al. 97a] A. Castro, J. Cossio, and John M. Neuberger. "A Sign-Changing Solution for a Superlinear Dirichlet Problem." Rocky Mountain J. of Math. 27:4 (1997), 1041-1053.

[Castro et al. 97b] A. Castro, J. Cossio, and John M. Neuberger. "On Multiple Solutions of a Nonlinear Dirichlet Problem." Nonlinear Analysis TMA 30:6 (1997), 36573662. 
[Castro et al. 99] A. Castro, J. Cossio, and John M. Neuberger. "A Minimax Principle, Index of the Critical Point, and Existence of Sign-Changing Solutions to Elliptic Boundary Value Problems." Electronic J. of Diff. Eq. 1998:2 (1998), 1-18.

[Castro et al. 03] A. Castro, P. Drabek, and John M. Neuberger, "A Sign-Changing Solution for a Superlinear Dirichlet Problem, II." In Proceedings of the Fifth Mississippi State Conference on Differential Equations and Computational Simulations (Mississippi State, MS, 2001), pp. 101-107 (electronic) Electron. J. Differ. Equ. Conf. 10 (2003).

[Cheng 99] Sui Sun Cheng, "Stability of Partial Difference Equations." In New developments in Difference Equations and Applications (Taipei, 1997), pp. 93-135. Amsterdam: Gordon and Breach, 1999.

[Cheng 00] Sui Sun Cheng. "Invitation to Partial Difference Equations." In Communications in Difference Equations (Poznan, 1998), pp. 91-106. Amsterdam: Gordon and Breach, 2000.

[Cheng and Lin 99] Sui Sun Cheng and Yi-Zhong Lin, "General Solutions of a Four-Point Partial Difference Equation." Far East J. Math. Sci. 1:4 (1999), 507-516.

[Cheng and Liu 97] Sui Sun Cheng and Shu Tang Liu. "Existence of Positive Solutions of a Partial Difference Equation." Far East J. Math. Sci. 5:3 (1997), 387-392.

[Cheng and Pao 02] Sui Sun Cheng and C. V. Pao. "Entire Solutions of Discrete Elliptic Equations." Southeast Asian Bull. Math. 26:2 (2002), 267-278.

[Choi and McKenna 93] Y. S. Choi and P. J. McKenna. "A Mountain Pass Method for the Numerical Solution of Semilinear Elliptic Problems." Nonlinear Analysis 20:4 (1993), 417-437.

[Chung 97] F. Chung, Spectral Graph Theory, CBMS Regional Conference Series in Mathematics, 92. Providence, RI: American Mathematical Society 1997.

[Cossio et al. 00] J. Cossio, S. Lee, and John M. Neuberger. "A Reduction Algorithm for Sublinear Dirichlet Problems." Nonlinear Analysis 47:5 (2001), 3379-3390.

[Costa et al. 01] D. Costa, Z. Ding, and John M. Neuberger. "A Numerical Investigation of Sign-Changing Solutions to Superlinear Elliptic Equations on Symmetric Domains." J. Comput. Appl. Math. 131 (2001), 299-319.

[Golubitsky et al. 88] M. Golubitsky, I. Stewart, and D. Schaeffer. Singularities and Groups in Bifurcation Theory. Vol. II, Applied Mathematical Sciences, 69. New York: Springer-Verlag, 1988.

[Kevrekidis et al. 02] P. G. Kevrekidis, I. G. Kevrekidis, A. R. Bishop, and E. S. Titi. "Continuum Approach to Discreteness." Phys. Rev. E (3) 65:4 (2002).

[Lehoucq et al. 90] R. B. Lehoucq, D. C. Sorensen, and C. Yang. ARPACK Users' Guide: Solution of Large-Scale Eigenvalue Problems with Implicitly Restarted Arnoldi Methods. Software, Environments, and Tools. Philadelphia: SIAM, 1998.
[Liu and Chen 04] Shu Tang Liu and Guanrong Chen. "Oscillations of Second-Order Nonlinear Partial Difference Equations." Rocky Mountain J. Math. 34:2 (2004), 699711.

[Liu et al. 00] Shu Tang Liu, Yongqing Liu, Xin-Ping Guan, Jun Yang, and Sui Sun Cheng. "Existence of Monotone Positive Solution of Neutral Partial Difference Equation." J. Math. Anal. Appl. 247:2 (2000), 384-396.

[Ljusternik and Schnirelmann 04] L. Ljusternik and L. Schnirelmann. Méthodes topologiques dans les problèmes variationels. Paris: Hermann, (1934).

[Marchuk 82] G. I. Marchuk. Methods of Numerical Mathematics. Second edition. Translated from the Russian by Arthur A. Brown. Applications of Mathematics, 2. New York: Springer-Verlag, 1982.

[Neuberger 97a] John M. Neuberger. "GNGA: Recent Progress and Open Problems for Semilinear Elliptic PDE." In Variational Methods: Open Problems, Recent Progress, and Numerical Algorithms, pp. 201-237, Contemp. Math., 357. Providence, RI: Amer. Math. Soc., 2004.

[Neuberger 97b] John M. Neuberger, "A Numerical Method for Finding Sign-Changing Solutions of Superlinear Dirichlet Problems." Nonlinear World 4:1 (1997), 73-83.

[Neuberger 97c] J. W. Neuberger. Sobolev Gradients and Differential Equations, Lecture Notes in Mathematics, 1670. Berlin: Springer-Verlag, 1997.

[Neuberger 98] John M. Neuberger. "A Sign-Changing Solution for a Superlinear Dirichlet Problem with a Reaction Term Nonzero at Zero." Nonlinear Anal. 33:5 (1998), 427441.

[Neuberger and Swift 01] John M. Neuberger and J. W. Swift. "Newton's Method and Morse Index for Semilinear Elliptic PDEs." Internat. J. Bifur. Chaos Appl. Sci. Eng. 11:3 (2001), 801-820.

[Neuberger et al. 03] John M. Neuberger, D. Rice, and J. W. Swift. "Numerical Solutions of a Vector Ginzburg-Landau Equation with a Triple Well Potential." Int. J. Bif./ and Chaos Appl. Sci. Engrg 13:11 (2003), 3295-3306.

[Neuberger et al. 05a] John M. Neuberger, N. Sieben, and J. W. Swift. "Computing Eigenfunctions on Koch's Snowflake: A New Grid and Symmetry." To appear in J. Comput. Appl. Math., 2005.

[Neuberger et al. 05b] John M. Neuberger, N. Sieben, and J. W. Swift. "Semilinear BVP on Koch's Snowflake: Symmetry and Automated Branch Following Using GNGA." Preprint, 2005.

[Pao 95] C. V. Pao. "Block Monotone Iterative Methods for Numerical Solutions of Nonlinear Elliptic Equations." $\mathrm{Nu}$ mer. Math. 72:2 (1995), 239-262.

[Pokornyı̆ and Pryadiev 04] Yu. V. Pokornyı̆ and V. L. Pryadiev. "Some Problems in the Qualitative Sturm-Liouville Theory on a Spatial Network" (Russian). Uspekhi Mat. Nauk 59:3 (2004), 115-150. 
[Rabinowitz 86] P. H. Rabinowitz. Minimax Methods in Critical Point Theory with Applications to Differential Equations, CBMS Regional Conference Series in Mathematics, 65. Providence, RI: American Mathematical Society, 1986.

[Strikwerda 89] John C Strikwerda. Finite Difference Schemes and Partial Differential Equations, The Wadsworth and Brooks/Cole Mathematics Series. Pacific Grove, CA: Wadsworth and Brooks/Cole, 1989.
[Zhang 05] Guang Zhang. "Existence of Nontrivial Solutions for Discrete Elliptic Boundary Value Problems." Preprint, 2005 .

[Zhang and Cheng 02] Guang Zhang and Sui Sun Cheng. "Eventually Positive Solutions of Nonlinear Neutral Difference Equations." Int. Math. J. 2:3 (2002), 265-278.

[Zhang and Cheng 06] Guang Zhang and Sui Sun Cheng. "Existence of Solutions for a Nonlinear System with a Parameter." J. Math. Anal. App. 314:1 (2006), 311-319.

John M. Neuberger, Department of Mathematics and Statistics, Box 5717, Northern Arizona University, Flagstaff, AZ 86011 (John.Neuberger@NAU.edu)

Received January 31, 2005; accepted in revised form October 29, 2005. 